\title{
Photoinversion Reaction of a Pyrenyl Sulfoxide in Solution and in Polymer Matrices
}

\author{
Yasuyuki Tsurutani, ${ }^{\dagger}$ Takashi Yamashita $^{\dagger}{ }^{\circ}$ and Kazuyuki HoriE \\ Department of Chemistry and Biotechnology, Graduate School of Engineering, The University of Tokyo, \\ 7-3-1 Hongo, Bunkyo-ku, Tokyo 113, Japan
}

(Received April 7, 1997)

\begin{abstract}
A pyrenyl-substituted chiral sulfoxide, $(R)-(+)$-1-pyrenyl 4-tolyl sulfoxide (R-TPYS) was synthesized and photoracemized in acetonitrile, benzene, toluene and chloroform. Quantum yields of their photoinversion reactions were $0.02-0.03$ from the changes in $\mathrm{CD}$ spectra and the rates of decomposition measured by high-performance liquid chromatography (HPLC) during photoirradiation. Quantum yields of photoinversion for R-TPYS in poly(methyl methacrylate) (PMMA) and in polystyrene were almost the same as in solution. It is considered that photoinversion reaction of R-TPYS is not affected by the polymer matrix at room temperature. The reaction at $88 \mathrm{~K}$ deviates from the first-order kinetics. The free volume around the sulfoxide in polymer matrix at $88 \mathrm{~K}$ does not seem to be enough compared to the sweep volume necessary for the reaction to occur.
\end{abstract}

KEY WORDS Pyrenyl-Substituted Sulfoxide / Sulfoxide / Photoinversion Reaction / Photoracemization / Chirality / Free Volume / Poly(methyl methacrylate) / Polystyrene /

Characteristic features of reactions in polymer solids are different from those in solutions. In polymer matrices the mobility of reactants is the dominant factor of the reaction. Because the local motion of the main chain and the free rotation of the side chain are not frozen below glass transition temperature, $T_{\mathrm{g}}$, of matrix polymers, intermolecular or intramolecular reactions can take place sometimes even at temperature below $T_{\mathrm{g} .}{ }^{1,2}$ Reaction rates in polymer solids are controlled by the balance of the molecular motion of a matrix, the free volume required for the reaction and inherent chemical reaction rates. Solid-state reactions in amorphous polymers proceed in three steps as the reaction temperature changes from high to low: (1) reaction rates are determined by chemical reaction paths and are the same as in solution, (2) reaction rates reflect molecular motion of the polymer matrix and proceed heterogeneously, (3) reactions cannot occur because of the absence of molecular motion. The size of the free volume required for reaction to occur determines which changes among the above steps are brought about at transition temperatures $\left(T_{\mathrm{g}}, T_{\beta}, T_{\gamma}\right)$ of the matrix polymer. For example, the isomerization of spirobenzopyran which requires large free volume during the reaction shows the change from (1) to (2) at $T_{\mathrm{g}}$ of matrix polymers. ${ }^{3,4}$ In the case of azobenzene which requires smaller free volume for isomerization compared to spirobenzopyran, reaction proceeds in step (1) at room temperature (below $T_{\mathrm{g}}$ ) and with decrease in temperature it shows changes from (1) to (2) together with clear deviation from the first-order kinetics. ${ }^{5-9}$ In the case of the photoinversion reaction of a sulfoxide, it might be expected that reaction proceeds similarly as in solution even in a polymer matrix with a small free volume, because photoinversion can be proceeded by the movement of the lone pair and oxygen atom.

Sulfoxide is widely applied to asymmetric synthesis as a chiral auxiliary. ${ }^{10}$ It is known that sulfoxides are stable at room temperature but thermal racemization occurs at

\footnotetext{
† To whom correspondence should be addressed.
}

about $200^{\circ} \mathrm{C}$ due to the pyramidal inversion. ${ }^{11}$ Studies of photochemistry of sulfoxides are mainly concerned with reactions induced by radical pair produced by the cleavage of S-C bonds. ${ }^{12-21}$ Systematic study of the triplet of sulfoxides has been made recently. ${ }^{22}$ There were some attempts at light induced asymmetric induction of sulfoxides in which a chiral sensitizer was used, but no remarkable results have been reported. ${ }^{23-26} \mathrm{Al}-$ though interest in asymmetric photochemical reactions is growing, ${ }^{27}$ little noticeable research on photochemistry of sulfoxides as chiral molecules has been made.

It was reported by Mislow et al., ${ }^{28-31}$ that aromatic chiral sulfoxides undergo photoracemization with sensitized or direct light irradiation, which is believed due to the photochemical pyramidal inversion of sulfoxides. But little further research on this reaction has been made in recent years. Consequently the detailed mechanisms of this inversion reaction are not known.

The pyramidal structure of sulfoxides with the sulfur atom in its center is shown in Figure 1. In the present study, a pyrenyl-substituted sulfoxide was synthesized for the first time. The $\mathrm{C}-\mathrm{S}$ bond of this sulfoxide cannot be cleaved easily because of direct bonding between aromatic carbon and sulfur atom. There are few sulfoxides which emit fluorescence at room temperature. ${ }^{22}$ Pyrenyl-substituted sulfoxide emits strong fluorescence. Longer wavelength absorption and strong fluorescence emission would make this molecule suitable for dynamics and excited singlet state measurements. In the present paper the pyrenyl sulfoxide was photoracemized in solution and in polymer matrices and the quantum yields of its photoinversion reaction were estimated and discussed in relation to the required free volume for the photoinversion reaction (sweep volume).

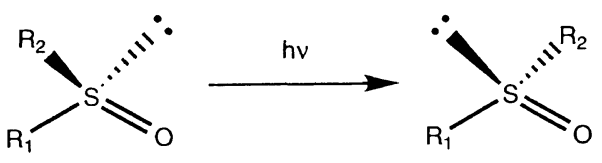

Figure 1. Photoinversion reaction of a sulfoxide 


\section{EXPERIMENTAL}

\section{Instrumentation}

All UV-visible absorption measurements were performed with a JASCO UVIDEC $660 \mathrm{UV} / \mathrm{VIS}$ spectrophotometer. IR spectra were obtained with a JASCO IR-700 infrared spectrophotometer. NMR spectra were measured with JEOL JNM-GX400 FT-NMR spectrometer. Luminescence spectra were recorded with a Hitachi 850 fluorescence spectrophotometer. Optical rotatory power was obtained with a JASCO DIP-360 digital polarimeter. $C D$ spectra were recorded with a JASCO J-500A spectropolarimeter. Melting points were measured with a Meihohsha sharp melting pointer. High-performance liquid chromatography (HPLC) was carried out with a JASCO UVIDEC 100-IV UV detector equipped with Wakosil 5SIL $(4.6 \mathrm{~mm} \times 200 \mathrm{~mm})$ column (Wako) and JASCO 807-IT integrator. Optical purity was measured with a Shimadzu SPD-6A UV detector and Hitachi D-2500 integrator with Daicel Chiralcel OD $(4.6 \mathrm{~mm} \times 250 \mathrm{~mm})$. All solvents and reagents were obtained from Tokyo Kasei and used without further purification, unless otherwise noted. In UV absorption and fluorescence measurements and photoracemization, all solvents were of spectrosol or luminasol grade (Wako) and used as received.

\section{Synthesis of $(R)-(+)-1-P y r e n y l$ 4-Tolyl Sulfoxide $(R-$ TPYS)}

R-TPYS was synthesized from the sulfinate with pyrenylmagnesium bromide by a method analogous to

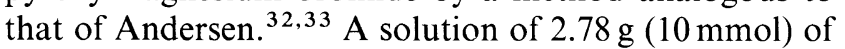
bromopyrene in $20 \mathrm{ml}$ anhydrous tetrahydrofuran (THF) was dropped into $0.315 \mathrm{~g}(13.8 \mathrm{mmol})$ of magnesium in $10 \mathrm{ml}$ THF under nitrogen atmosphere. After reflux for $1 \mathrm{~h}$, the mixture was cooled to $-78^{\circ} \mathrm{C}$ and a solution of $2.95 \mathrm{~g}(10 \mathrm{mmol})$ of $(1 S, 2 R, 5 S)-(+)$-menthyl- $(R)-p$ toluenesulfinate $\left([\alpha]_{\mathrm{D}}^{20}+201.0^{\circ}, c 2.0 \mathrm{~g} \mathrm{dl}^{-1}\right.$, acetone $)$ in $20 \mathrm{ml}$ THF was added dropwise for $2 \mathrm{~h}$ with stirring and cooling in an ice bath. $30 \mathrm{ml}$ of aqueous ammonium chloride $(1.25 \mathrm{M})$ were added. After stirring for $15 \mathrm{~min}$, the THF solution was removed by decanting. The water layer was extracted with dichloromethane. A mixture of THF and dichloromethane solution was evaporated, dissolved again in dichloromethane and dried over anhydrous sodium sulfate. The product was isolated by column chromatography on silica gel (Wako Gel C-200, eluent benzene: $\mathrm{THF}=20: 1$ ) and purified by recrystallization in benzene and hexane. $0.527 \mathrm{~g}$ of a yellow crystal were obtained with a $15.5 \%$ yield. Melting point was $153-155^{\circ} \mathrm{C}$. The specific rotation: $[\alpha]_{\mathrm{D}}^{25}+1.05 \times 10^{3 \circ}$ ( $c=1.00 \mathrm{~g} \mathrm{dl}^{-1}$, dichloromethane). Enanthiomer excess: $98.8 \%$ (determined by chiral HPLC column, eluent hexane: isopropyl alcohol $=9: 1$, flow rate $0.5 \mathrm{ml} \mathrm{min}^{-1}$, monitored at $254 \mathrm{~nm}$ ).

IR (KBr): $v 1046 \mathrm{~cm}^{-1}(\mathrm{~S}=\mathrm{O}), 1593,1493 \mathrm{~cm}^{-1}(\mathrm{ar}-$ omatic $\mathrm{C}=\mathrm{C}), 3042 \mathrm{~cm}^{-1}$ (aromatic $\left.\mathrm{C}-\mathrm{H}\right) .{ }^{1} \mathrm{H}$ NMR $\left(\mathrm{CDCl}_{3}\right): \delta 7.2,7.6(\mathrm{~d}, 4 \mathrm{H}$, tolyl- $\underline{\mathrm{H}}), 8.1-8.6(\mathrm{~m}, 9 \mathrm{H}$, pyrenyl- $-\underline{\mathrm{H}}), 2.3\left(\mathrm{~s}, 3 \mathrm{H}, \mathrm{Ar}-\mathrm{C}-\mathrm{H}_{3}\right)$. Elemental Analysis Calcd for $\mathrm{C}_{23} \mathrm{H}_{16} \mathrm{OS}$ : C, 81.14\%; H, 4.74\%; S, 9.42\%. Found: $\mathrm{C}, 81.20 \%$; H, 5.01\%; S, 9.50\%. CD: $\lambda_{\max }(\Delta \varepsilon)$ (acetonitrile solution): 221 (6.9), $232(-6.0), 246$ (24.6), 277 (-19.4), 303 (6.4), 333 (14.1), 349 (15.9), 375 (1.3).

\section{Photoracemization in Solution}

Solutions of $2.5 \mathrm{ml}$ of $1 \times 10^{-5}-2 \times 10^{-5} \mathrm{M}$ R-TPYS in acetonitrile, benzene, toluene and chloroform were irradiated with $365 \mathrm{~nm}$ light from a $250-\mathrm{W}$ high pressure mercury lamp (Ushio USH-250D) with a combination of interference filter (Toshiba, KL-36) and color filter (Toshiba, UV-25). Ellipticity (optical activity) changes were traced with CD spectra. Irradiation was continued until peaks of CD spectra disappeared. Light intensity before and after irradiation was measured at $436 \mathrm{~nm}$ using an interference filter (Toshiba KL-44) and color filter (Toshiba L-39) with an Advantest TQ8210 optical powermeter calibrated by chemical actinometry (potassium ferrioxalate actinometry). UV spectra before and after photoracemization were obtained to check for by-products. Aliquots of solution during irradiation $(0.5 \mathrm{ml})$ were sampled. Solutions during and after irradiation were quantified by HPLC to determine the rates of decomposition of TPYS during photoracemization.

\section{Photoracemization in Polymer Matrices}

$1 \mathrm{~mm}$ thick polymer films containing R-TPYS $(0.80 \times$ $\left.10^{-4}-1.0 \times 10^{-4} \mathrm{M}\right)$ on quartz plates were prepared using the solvent-cast method and irradiated with $365 \mathrm{~nm}$ light from the high pressure mercury lamp in the same way as for the solution.

\section{Photoracemization in Polymer Matrices at a Low Temperature \\ Poly(methyl methacrylate) (PMMA) films containing} R-TPYS $\left(9.79 \times 10^{-5} \mathrm{M}\right)$ prepared by the solvent-cast method were set in the cryostat (Oxford DN1754), cooled to $88 \mathrm{~K}$ and irradiated at $365 \mathrm{~nm}$ with the high pressure mercury lamp.

\section{RESULTS AND DISCUSSION}

\section{Characterization of $R-T P Y S$}

Chiral sulfoxides are synthesized by allowing opticallyactive sulfinate esters to react with Grignard reagents via an inversion mechanism. ${ }^{32,33}$ Therefore the synthesized chiral sulfoxide has $R$ configuration. Optical rotation power, $[\alpha]_{\mathrm{D}}^{25}$, of the synthesized R-TPYS was $+1.05 \times$ $10^{3 \circ}$ at the sodium D line. Sulfoxides of $R$ configuration have positive rotation except for only a few cases. ${ }^{33}$

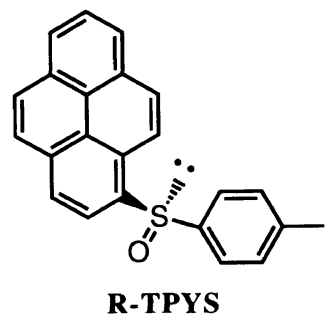

The absorption spectra of R-TPYS in acetonitrile are shown in Figure 2 (solid line). Absorption of the pyrenyl group is observed at $280 \mathrm{~nm}, 340 \mathrm{~nm}$ and $352 \mathrm{~nm}$. New weak absorption is observed in the longest wavelength region at $380 \mathrm{~nm}$. 0-0 transition between ground and excited singlet states for pyrene $\left(26900 \mathrm{~cm}^{-1} ; 371 \mathrm{~nm}\right)$ is not permitted by selection rules because of the same 


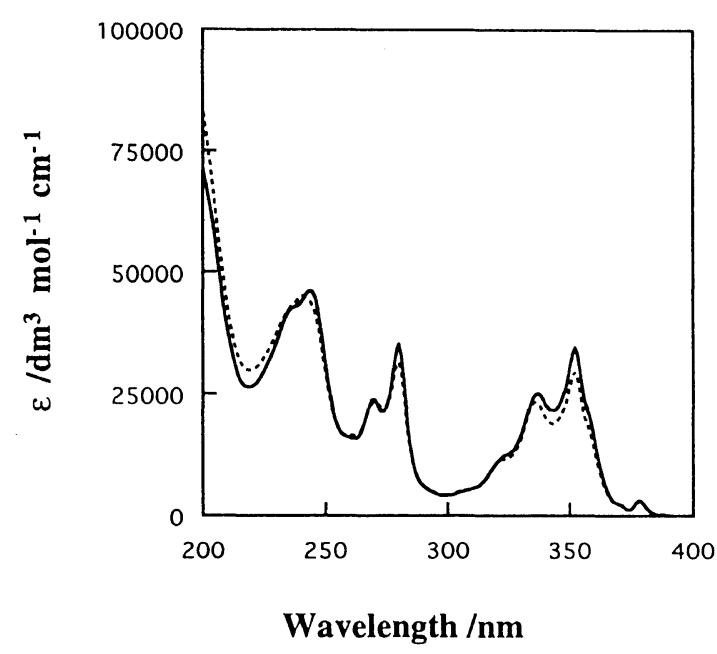

Figure 2. UV spectra of R-TPYS in acetonitrile (solid line: before irradiation; dotted line: after irradiation for $5520 \mathrm{~s}$ ).

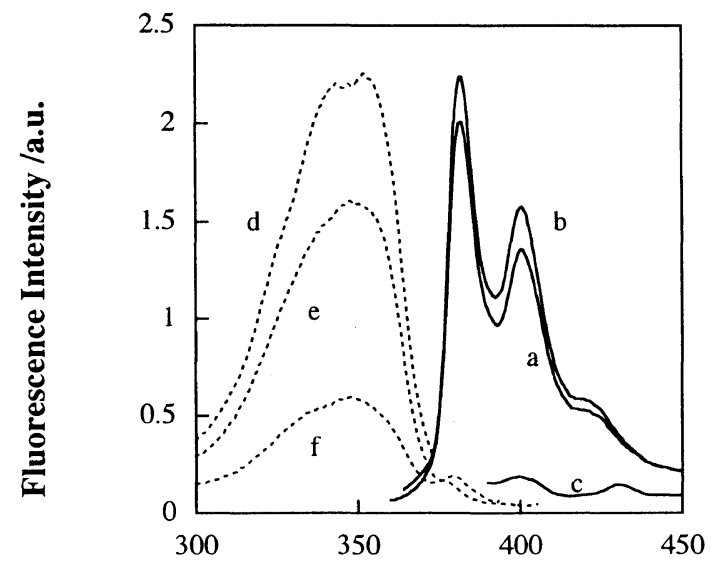

Wavelength /nm

Figure 3. Fluorescence spectra of R-TPYS in benzene $\left(1.12 \times 10^{-5}\right.$ M). (solid lines: emission spectra exited at $340 \mathrm{~nm}$ (a), $354 \mathrm{~nm}$ (b), and $380 \mathrm{~nm}$ (c); dotted lines: excitation spectra monitored at $382 \mathrm{~nm}$ (d), $402 \mathrm{~nm}$ (e), and $425 \mathrm{~nm}(\mathrm{f}))$.

symmetry of electronic states. ${ }^{34,35}$ Distortion of symmetric electronic states of the sulfoxide is supposed to loosen the symmetry-forbiddenness, so it produce new absorption at $380 \mathrm{~nm}$. The absorption spectra were measured for R-TPYS in various solvents such as acetonitrile, hexane, benzene, dichloromethane, methanol, and iso-butyl alcohol, and absorption peaks were observed to red-shift $(0-4 \mathrm{~nm})$ at $340 \mathrm{~nm}, 350 \mathrm{~nm}$, and $380 \mathrm{~nm}$ absorption in polar solvents. Therefore these are $\pi-\pi^{*}$ absorptions of the pyrenyl group.

Fluorescence spectra of R-TPYS in benzene excited at $340 \mathrm{~nm}, 354 \mathrm{~nm}$, and $380 \mathrm{~nm}$ are shown in Figure 3. Almost the same emission spectra were observed for $340 \mathrm{~nm}, 354 \mathrm{~nm}$, and $380 \mathrm{~nm}$ excitation. The solvent effects are also observed. The peaks of fluorescence red-shifted somewhat $(2-3 \mathrm{~nm})$ in polar solvents. This solvent effect on fluorescence spectra does not contradict the case for absorption spectra.

\section{Photoracemization in Solution}

The change in CD spectra of R-TPYS in acetonitrile during photoirradiation at $365 \mathrm{~nm}$ is shown in Figure 4. The decrease in optical activity in solution caused by

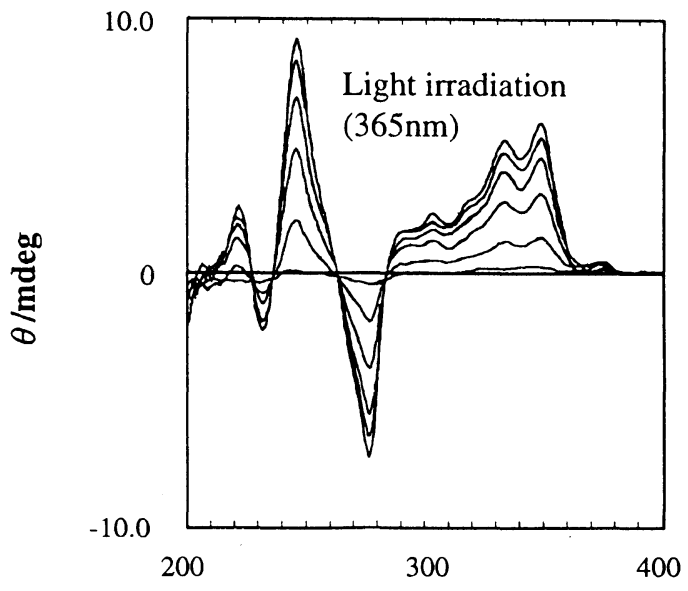

Wavelength /nm

Figure 4. Change in CD spectra of R-TPYS in acetonitrile $\left(1.13 \times 10^{-5} \mathrm{M}\right)$ during photoirradiation at $365 \mathrm{~nm}$ (irradiation time: $0 \mathrm{~s}, 180 \mathrm{~s}, 480 \mathrm{~s}, 1200 \mathrm{~s}, 2640 \mathrm{~s}, 5520 \mathrm{~s}$ from the top).

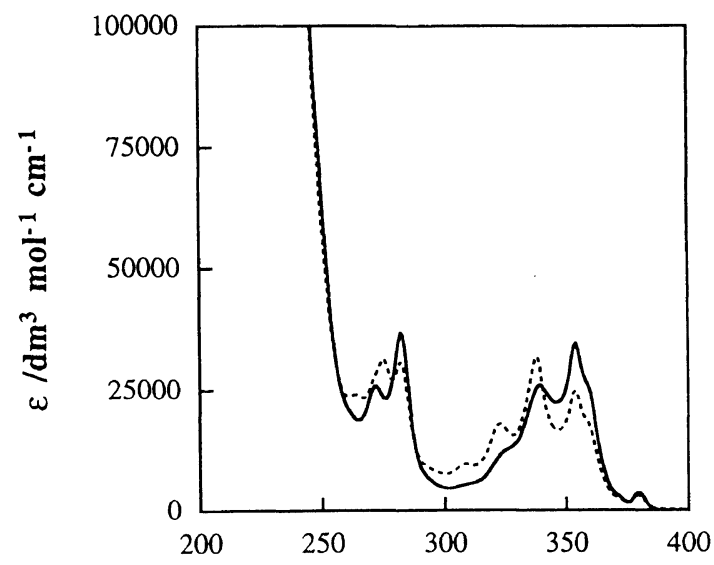

Wavelength /nm

Figure 5. UV spectra of R-TPYS in chloroform (solid line: before irradiation; dotted line after irradiation for $5580 \mathrm{~s}$ ).

photoracemization was recognized. Almost the same changes in $\mathrm{CD}$ spectra were recognized for four solvents (acetonitrile, benzene, toluene, and chloroform). As is shown in Figure 2, there was some change in UV spectra of acetonitrile and benzene solutions after irradiation. Changes were recognized in UV spectra of toluene and chloroform solutions after irradiation as are shown in Figure 5, suggesting that some by-products exist in toluene and chloroform solution.

The rates of photoinversion are expressed in eq $1-5$,

$$
\begin{gathered}
-\frac{\mathrm{d} C_{R}(t)}{\mathrm{d} t} \cdot S \cdot l \times 10^{-3}=\left(A_{R}(t) \cdot \Phi-A_{S}(t) \cdot \Phi\right) S \\
-\frac{\mathrm{d} C_{S}(t)}{\mathrm{d} t} \cdot S \cdot l \times 10^{-3}=\left(A_{S}(t) \cdot \Phi-A_{R}(t) \cdot \Phi\right) S \\
A_{R}(t)=I_{0} \cdot\left(1-10^{-O D}\right) \cdot \frac{C_{R}(t)}{C_{0}} \\
A_{S}(t)=I_{0} \cdot\left(1-10^{-O D}\right) \cdot \frac{C_{S}(t)}{C_{0}}
\end{gathered}
$$




$$
C_{R}(t)-C_{S}(t)=C_{0} \cdot \exp \left(-\frac{2 \cdot I_{0}\left(1-10^{-o D}\right) \Phi \times 10^{3}}{C_{0} \cdot l} \cdot t\right)
$$

where $I_{0}$ is the number of irradiated photons per unit time and unit cross section, $S$ is the area of the irradiated cross section, $C_{R}(t)$ and $C_{S}(t)$ are concentration of R-TPYS and S-TPYS $((S)-(-)-1$-pyrenyl 4-tolyl sulfoxide), respectively, and functions of time, $\varepsilon$ is the molar extinction coefficient of R- and S-TPYS, $l$ is the thickness of system, $A_{R}(t)$ and $A_{S}(t)$ are the numbers of photons per unit time and unit cross section which R-TPYS and S-TPYS absorb, respectively, $\Phi$ is the quantum yield of photoinversion reaction and $t$ is time. It is obvious that the quantum yields of photoinversion reaction for R-TPYS and S-TPYS are equal because of symmetry.

Ellipticity at $349 \mathrm{~nm}, \theta(t)$, is expressed by eq 6 . The quantum yield of photoinversion reaction $\Phi$ is obtained from changes in $\theta(t)$ by eq 12 .

$$
\begin{gathered}
\theta=33 \times \Delta O D \\
\Delta \varepsilon=\varepsilon_{l R}-\varepsilon_{r R}=\varepsilon_{r S}-\varepsilon_{l S} \\
O D_{l}(t)=\left\{\varepsilon_{l R} \cdot C_{R}(t)+\varepsilon_{l S} \cdot C_{S}(t)\right\} l \\
O D_{r}(t)=\left\{\varepsilon_{r R} \cdot C_{R}(t)+\varepsilon_{r S} \cdot C_{S}(t)\right\} l \\
\Delta O D(t)=O D_{l}(t)-O D_{r}(t)=\Delta \varepsilon\left(C_{R}(t)-C_{S}(t)\right) l \\
C_{R}(t)-C_{S}(t)=2 C_{R}(t)-C_{0}=\frac{\Delta O D(t)}{\Delta \varepsilon \cdot l}=\frac{\theta(t)}{33 \cdot \Delta \varepsilon \cdot l} \\
\ln \left(\frac{\theta(t)}{\theta_{0}}\right)=-\frac{2 \cdot I_{0}\left(1-10^{-O D}\right) \times 10^{3}}{C_{0} \cdot l} \Phi \cdot t=-A \cdot \Phi \cdot t
\end{gathered}
$$

where $\varepsilon_{l R}$ and $\varepsilon_{r R}$ are the molar absorptivities of R-TPYS for left-circularly-polarized light (1-CPL) and rightcircularly-polarized light (r-CPL) and $\varepsilon_{l S}$ and $\varepsilon_{r S}$ are also molar absorptivities of S-TPYS for 1-CPL and r-CPL, respectively.

The wavelength distribution of irradiated light intensity was calculated from the wavelength distribution of mercury lamp intensity, wavelength dependence of the sensitivity of the optical powermeter and transmittance of the filters. Absolute light intensity for each wavelength was calculated from the wavelength distribution of the incident light and light intensity measured with optical powermeter, and converted to the number of irradiated photon, $I_{0}$, per unit time and unit cross section. Optical density, $O D$, was measured from UV spectra before irradiation. Parameter, $A$, was obtained from $O D, I_{0}, C_{0}$ and $l$.

First-order plots of the ellipticity, $\theta(t)$, against time in acetonitrile are shown in Figure 6. The quantum yields of photoinversion reaction, $\Phi$, were calculated from the slopes of the first-order plots. The values of $\Phi$ in four solvents are given in Table I. Experimental error of $\Phi$ was within $10 \%$.

Although side reactions are ignored in the calculations, it is possible that optical activity in solution decreased also owing to side reactions. The loss of TPYS in solution

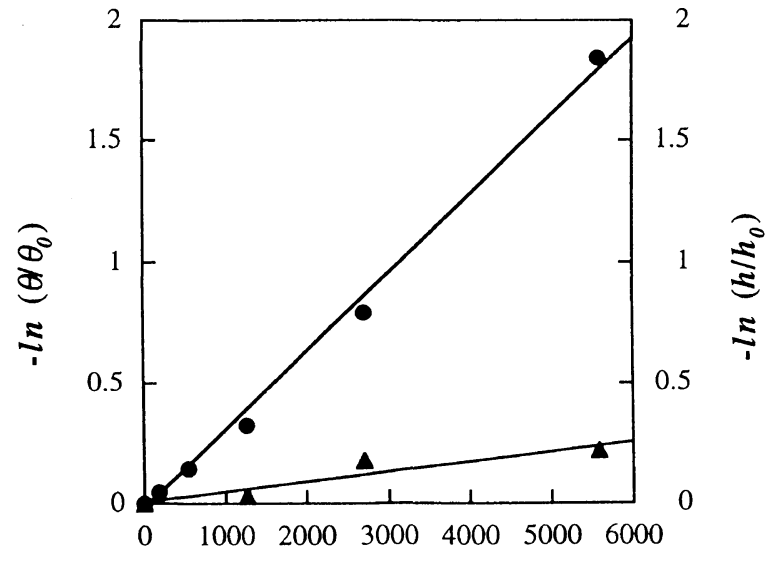

\begin{tabular}{|c|c|c|c|}
\hline \multirow{2}{*}{ Solvent } & \multicolumn{3}{|c|}{ Quantum yields } \\
\hline & $\Phi$ & $\Phi_{\mathrm{dec}}$ & $\Phi_{\text {inv }}$ \\
\hline Acetonitrile & $2.6 \times 10^{-2}$ & $4.4 \times 10^{-3}$ & $2.4 \times 10^{-2}$ \\
\hline Benzene & $2.8 \times 10^{-2}$ & $5.4 \times 10^{-3}$ & $2.5 \times 10^{-2}$ \\
\hline Toluene & $3.0 \times 10^{-2}$ & $4.9 \times 10^{-3}$ & $2.8 \times 10^{-2}$ \\
\hline Chloroform & $3.0 \times 10^{-2}$ & $4.4 \times 10^{-3}$ & $2.8 \times 10^{-2}$ \\
\hline
\end{tabular}

Irradiation time /s

Figure 6. First-order plots for photoracemization of R-TPYS in acetonitrile at room temperature $\left(\mathbf{O},-\ln \left(\theta(t) / \theta_{0}\right) ; \mathbf{\Lambda},-\ln \left(h(t) / h_{0}\right)\right)$.

Table I. Quantum yields of photoinversion reactions, $\Phi$, photolysis, $\Phi_{\text {dec }}$, and net inversion, $\Phi_{\text {inv }}$, for R-TPYS in acetonitrile, benzene, toluene, and chloroform at room temperature. Concentrations are $1-2 \times 10^{-5} \mathrm{M}$

during light irradiation was measured by HPLC. The ratio of decomposition is less than $20 \%$ after 92 min of irradiation in acetonitrile solution. The quantum yields of photolysis of TPYS, $\Phi_{\text {dec }}$, were obtained from the first-order plots (Figure 6) of the peak area (expressed as $h(t))$ of TPYS and light intensity. Experimental error of $\Phi_{\text {dec }}$ was within $20 \%$. Since decrease in optical activity during light irradiation was induced also by the loss of TPYS, the net quantum yields of photoinversion, $\Phi_{\text {inv }}$, were recalculated from $\Phi$ and $\Phi_{\text {dec }}$ by using eq 13 and are listed in Table I,

$$
2 \Phi=2 \Phi_{\mathrm{inv}}+\Phi_{\mathrm{dec}}
$$

where $2 \Phi$ is equal to quantum efficiency of loss of optical activity, $2 \Phi_{\mathrm{inv}}$ is equal to that of photoracemization induced by net photoinversion. It seems that neither $\Phi_{\text {inv }}$ nor $\Phi_{\mathrm{dec}}$ are affected by solvent (Table I). $\Phi_{\mathrm{inv}}$ and $\Phi_{\mathrm{dec}}$ are estimated to be $0.02-0.03$ and $0.004-0.005$, respectively, irrespective of the solvent.

The photolysis of sulfoxides has been investigated in detail. ${ }^{12-21}$ According to these reports, it is supposed that side reactions are initiated by $\mathrm{C}-\mathrm{S}$ bond cleavage and radical formation. The UV spectra of acetonitrile and benzene solution after irradiation changed very little but changes were recognized for toluene and chloroform solution. It seems that differences in UV spectra of TPYS after light irradiation are due to those in by-products produced by the photolysis.

\section{Photoracemization in Polymer Matrices}

The peak of CD spectra, $\theta(t)$, decreased during 


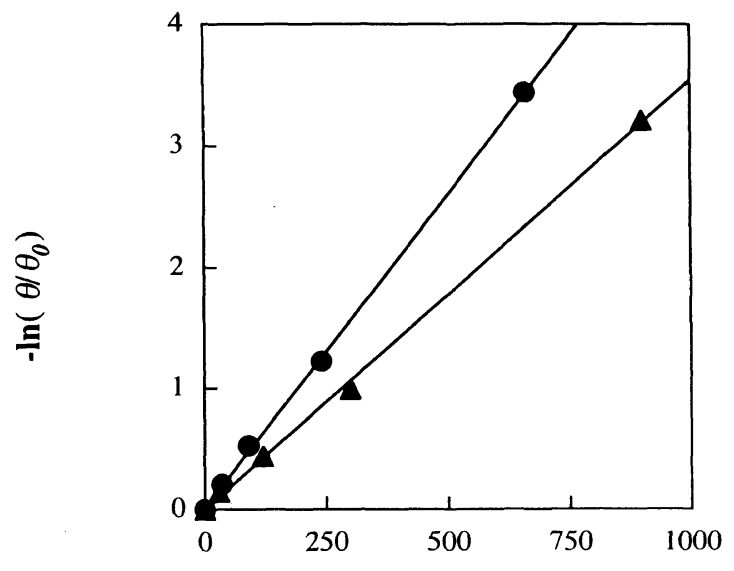

Irradiation time /s

Figure 7. First-order plots for photoracemization of R-TPYS in polystyrene $(\bullet)$ and PMMA $(\boldsymbol{\Delta})$ at room temperature.

Table II. Quantum yields of photoinversion reactions, $\Phi$, for R-TPYS in PMMA and polystyrene

\begin{tabular}{cccc}
\hline Polymer & Temperature & $\begin{array}{c}\text { Concentration/ } \\
\mathrm{M}\end{array}$ & Quantum yields \\
\hline PMMA & r.t. & $9.79 \times 10^{-5}$ & $2.7 \times 10^{-2}$ \\
PMMA & $88 \mathrm{~K}$ & $9.79 \times 10^{-5}$ & $\begin{array}{c}1.6 \times 10^{-2} \\
(\text { Initial stage }) \\
\text { Polystyrene }\end{array}$ \\
& r.t. & $8.64 \times 10^{-5}$ & $3.0 \times 10^{-2}$ \\
\hline
\end{tabular}

photoirradiation at $365 \mathrm{~nm}$ to polymer films (polystyrene and PMMA) doped with R-TPYS at room temperature. UV spectra did not change after photoracemization. Although the rates of photolysis of TPYS in polymer films could not be measured, the rates of photolysis should not be larger than in solution. Similarly to the photoinversion in solution, straight lines were obtained for the first-order plots of $\theta(t)$ against time as shown in Figure 7, and quantum yields of photoinversion were determined from the slopes of the first-order plots. The quantum yields, $\Phi$, are $2.7 \times 10^{-2}$ and $3.0 \times 10^{-2}$ for R-TPYS in PMMA and polystyrene, respectively, at room temperature (Table II). These value were almost the same as in solution $(0.02-0.03)$. It is supposed that photoinversion reaction occurs in polymer films at room temperature with the same efficiency as in solution. The effect of polymer matrix was not observed. Photochemical reactions in polymer solids at a temperature below $T_{\mathrm{g}}$ are usually observed to deviate drastically from first-order kinetics. ${ }^{2}$ Present results may be due to the small sweep volume required for the photoinversion, compared to the free volume expanded by thermal fluctuation in these polymer matrices at room temperature.

\section{Photoracemization in Polymer Matrix at a Low Tem- perature}

A PMMA film doped with R-TPYS was refrigerated to $88 \mathrm{~K}$, and irradiated at $365 \mathrm{~nm} . \theta(t)$ of the sample decreased during light irradiation for $5 \mathrm{~h}$, and peaks of CD spectra disappeared. UV spectra of the sample did not change after $50 \mathrm{~min}$ irradiation and slightly changed after $5 \mathrm{~h}$ irradiation. For $50 \mathrm{~min}$ irradiation,

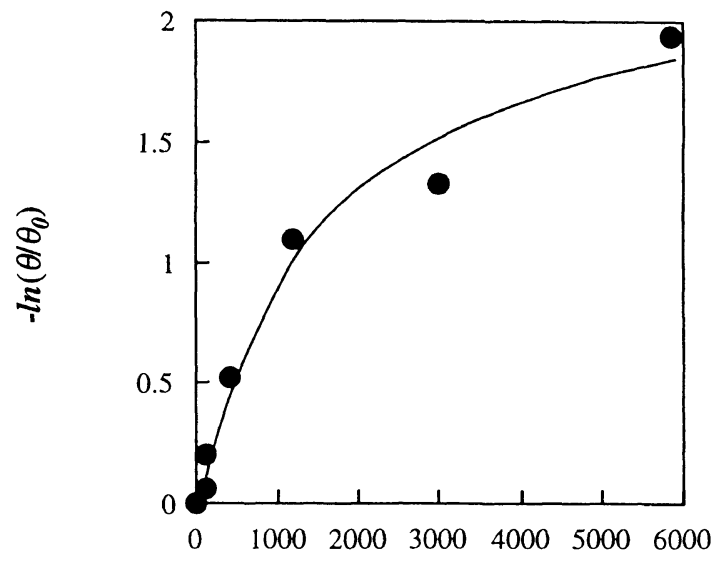

Irradiation time /s

Figure 8. First-order plots for photoracemization of R-TPYS in PMMA at $88 \mathrm{~K}$.

optical activity of the sample dramatically decreased. The decrease in $\theta(t)$ and no change of UV spectra mean that photoracemization proceeds even at $88 \mathrm{~K}$ in PMMA matrix.

The reaction deviates from the first-order kinetics as shown in Figure 8. This agrees with our previous results $^{5-9}$ for the photoisomerization of azobenzene, azonaphthalene, etc. in various polymer matrices. The quantum yield for the initial stage of the photoinversion reaction at $88 \mathrm{~K}$ is estimated to be 0.016 from the initial slope of the first-order plots and plots for conversion above $20 \%\left(\ln \theta / \theta_{0}=0.223\right)$ begin to deviate from a straight line. The obtained initial quantum yield is much larger than that of photolysis at room temperature. Consequently, photoinversion reaction proceeds in PMMA at $88 \mathrm{~K}$. Since there is distribution of free volume in polymer matrices, molecules in amorphous polymer solids react when (1) there is enough free volume around reaction sites and reactions proceed freely as in solution, (2) free volume is not so enough and reactions are controlled by molecular motions of polymer chains, (3) free volume is smaller than sweep volume and reactions cannot occur. Since the thermal fluctuation of polymer chains is restricted and the number of sites where TPYS cannot freely undergo inversion increases at low temperatures, first-order plots deviate from a straight line.

\section{CONCLUSION}

R-TPYS was photoracemized in acetonitrile, benzene, toluene, and chloroform solutions. Quantum yields of the photoinversion reaction were $0.02-0.03$ from changes of the CD spectra, $\theta(t)$, and the rates of photolysis obtained by HPLC during photoirradiation. Quantum yields of photolysis were estimated to be $0.004-0.005$ and one order of magnitude smaller than that of photoinversion. Although the rate of photolysis in polymer matrices was not measured, quantum yields of photoinversion for the samples in PMMA and in polystyrene seem to be almost the same as in solution. The photoinversion reaction of R-TPYS proceeds linearly based on the first-order kinetics and is not affected by 
the polymer matrices at room temperature. The reaction at $88 \mathrm{~K}$ deviates from the first-order kinetics above $20 \%$ conversion. The restriction of chain mobility at $88 \mathrm{~K}$ decreases the number of sites which have sufficiently large free volume around sulfoxides in the polymer matrix, compared to the size of sweep volume for the reaction to occur, and hence photoinversion is controlled by the local chain motion in the intermediate stage of photoracemization at $88 \mathrm{~K}$.

Acknowledgments. The authors are grateful to Prof. K. Saigo, Dr. Y. Hashimoto, and Dr. M. Hayashi for assistance in determining enantiomer excess, and to Dr. S. Machida for helpful discussion.

\section{REFERENCES}

1. G. Smets, Adv. Polym. Sci., 50, 17 (1983).

2. K. Horie and I. Mita, Adv. Polym. Sci., 88, 77 (1989).

3. K. Horie, M. Tsukamoto, and I. Mita, Eur. Polym. J., 21, 805 (1985)

4. K. Horie, K. Hirao, N. Kenmochi, and I. Mita, Makromol. Chem., Rapid Commun. 9, 267 (1988).

5. I. Mita, K. Horie, and K. Hirao, Macromolecules, 22, 558 (1989).

6. T. Naito, K. Horie, and I. Mita, Eur. Polym. J., 26, 1295 (1990).

7. T. Naito, K. Horie, and I. Mita, Macromolecules, 24, 2907 (1991).

8. T. Naito, K. Horie, and I. Mita, Polym. J., 23, 809 (1991).

9. T. Naito, K. Horie, and I. Mita, Polymer, 34, 4140 (1993).

10. M. C. Carreño, Chem. Rev., 95, 1717 (1995).

11. D. R. Rayner, A. J. Gordon, and K. Mislow, J. Am. Chem. Soc., 90(18), 4854 (1968).

12. A. G. Schultz, C. D. DeBoer, and R. H. Schlessinger, J. Am. Chem. Soc., 90(19), 5314 (1968).

13. A. G. Schultz and R. H. Schlessinger, J. Chem. Soc., Chem. Commun., 1483 (1969).

14. A. G. Schultz and R. H. Schlessinger, J. Chem. Soc., Chem. Commun., 1294 (1970)
15. B. S. Larsen, J. Kolc, and S. O. Lawesson, Tetrahedron, 27, 5163 (1971).

16. G. M. Gurria and G. H. Posner, J. Org. Chem., 38, 2419 (1973).

17. K. A. Muszkat, K. Praefcke, I. Khait, and R. Lüdersdorf, $J$. Chem. Soc., Chem. Commun., 898 (1979).

18. I. Khait, R. Lüdersdorf, K. A. Muszkat, and K. Praefcke, J. Chem. Soc., Perkin Trans. 2, 1417 (1981).

19. B. C. Gilbert, B. Gill, and M. D. Sexton, J. Chem. Soc., Chem. Commun., 78 (1978)

20. C. Chatgilialoglu, B. C. Gilbert, B. Gill, and M. D. Sexton, J. Chem. Soc., Perkin Trans. 2, 1141 (1980).

21. Y. Guo and W. S. Jenks, J. Org. Chem,. 62, 857 (1997).

22. W. S. Jenks, W. Lee, and D. Shutters, J. Phys. Chem., 98, 2282 (1994).

23. G. Balavoine, S. Juge, and H. B. Kagan, Tetrahedron Lett., 42, 4159 (1973).

24. W. H. Pirkle and P. L. Rinaldi, J. Am. Chem. Soc., 99(10), 3510 (1977).

25. C. Eskenazi, J. F. Nicoud, and H. B. Kagan, J. Org. Chem., 44(6), 995 (1979).

26. L. Finzi, G. Maccagnani, S. Masiero, B. Samori, and P. Zani, Liq. Crystals, 6(2), 199 (1989).

27. Y. Inoue, Chem. Rev., 92, 741 (1992).

28. K. Mislow, M. Axelrod, D. R. Rayner, H. Gotthardt, L. M. Coyne, and G. S. Hammond, J. Am. Chem. Soc., 87(21), 4958 (1965).

29. G. S. Hammond, Hans Gotthardt, L. M. Coyne, M. Axelrod, D. R. Rayner, and K. Mislow, J. Am. Chem. Soc., 87(21), 4959 (1965).

30. R. S. Cooke and G. S. Hammond, J. Am. Chem. Soc., 90(11), 2958 (1968).

31. R. S. Cooke and G. S. Hammond, J. Am. Chem. Soc., 92(9), 2739 (1970).

32. K. K. Andersen, Tetrahedron Lett., 3, 93 (1962).

33. K. K. Andersen, W. Gaffield, N. E. Papanikolaou, J. W. Foley, and R. I. Perkins, J. Am. Chem. Soc., 86, 5637 (1964).

34. J. B. Birks, "Photophysics of Aromatic Molecules," WileyInterscience, London, 1970, Chapter 1, pp 8-10.

35. J. B. Birks, "Photophysics of Aromatic Molecules," WileyInterscience, London, 1970, Chapter 3, p 71 